\title{
Understanding Microwave-assisted Fixation: Energy Versus Heating
}

\section{R.T. Giberson}

Research and Development, Ted Pella, Inc., Redding, CA 96003

The ability to control all aspects of the microwave environment during processing has been an elusive goal since the first report of microwave-assisted methods being used in the laboratory [1]. Recently, complete control of the microwave environment has been achieved through improved technology [2]. This evolution has made it possible to evaluate whether there is a potential microwave effect operating during processing [2]. Microwave induced sample heating has been cited as the chief or only mechanism to explain microwave-accelerated processes [3-5]. Older equipment and accessories offered minimal control of the microwave environment [3-6]. Up until recently the two controllable parameters in microwave processing have been time and sample temperature [2-8]. The recent development of equipment that can control processing temperatures without disruption of the microwave power output has made it possible to evaluate the role of microwave energy in processing [2].

The role of the microwave and its effect on microwave-assisted chemical fixation has been described best for electron microscopy [8]. In that report the technology and accessories were adequate to control the microwave environment for the time periods described. Thus, the processing conditions could be easily reproduced on succeeding runs. The report also demonstrated that small tissue pieces are rapidly infiltrated with fixatives, solvents and resins in a microwave environment fitted with vacuum processing capabilities and variable wattage.

Control of the microwave environment, however, becomes difficult when the processing times extend beyond a few minutes. Reagent temperatures rise to preset maximums and activate temperature control devices, stopping the continuous output of microwave irradiation. The majority of microwave systems available today operate in this manner, making precise control of the microwave environment difficult or impossible. The problem has been resolved with new technology that can deliver continuous microwave energy at fixed temperatures as low as $4^{\circ} \mathrm{C}$. In a recent paper reporting on microwave-accelerated EDTA decalcification of bone a fixed temperature of $20^{\circ} \mathrm{C}$ was maintained for both the control and microwave processed samples [2]. These findings indicate the significance of microwave energy to microwave-accelerated processes.

This new technology has shown great promise for microwave-accelerated formaldehyde fixation of fresh tissue (research in progress). The mouse kidney shown in Figure 1 was fixed for 24 hours in modified Karnovsky's (2\% paraformaldehyde- $2.5 \%$ glutaraldehyde- $0.06 \mathrm{M}$ phosphate buffer, $\mathrm{pH}$ 7.2) followed by 1 hour in osmium tetroxide. Figure 2 is the same tissue and fixatives processed using microwave methods that required less than 10 minutes to complete both steps.

Recent findings indicate that a controlled microwave environment will reduce fixation and processing times for light or electron microscopy by over $90 \%$ compared to conventional methods and yield high quality results and images in the process $[2,7,8]$. 
[1] C.P. Mayers, J. Clin. Pathol. 23 (1970) 273.

[2] S.P. Tinling et al., Microsc. Microanal. 8 (suppl. 2) (2002) 148.

[3] L.P. Kok and M.E. Boon, Microwave Cookbook for Microscopists, $3^{\text {rd }}$ ed., Coulomb Press, Leyden, 1992.

[4] M.E. Boon and L.P. Kok, Micron. 25 (1994) 151.

[5] L.P. Kok et al., Scanning, 15 (1993) 100.

[6] G.R. Login and A.M. Dvorak, The Microwave Tool Book - A Practical Guide for Microscopists, Beth Israel Hospital, 1994.

[7] R.T. Giberson and D.E. Elliott, In: Microwave Techniques and Protocols, R.T. Giberson and R.S. Demaree Jr., eds. Humana Press, Totowa, NJ, 2001.

[8] R.T. Giberson, Microsc. Microanal. 7 (suppl. 2) (2001) 1192.

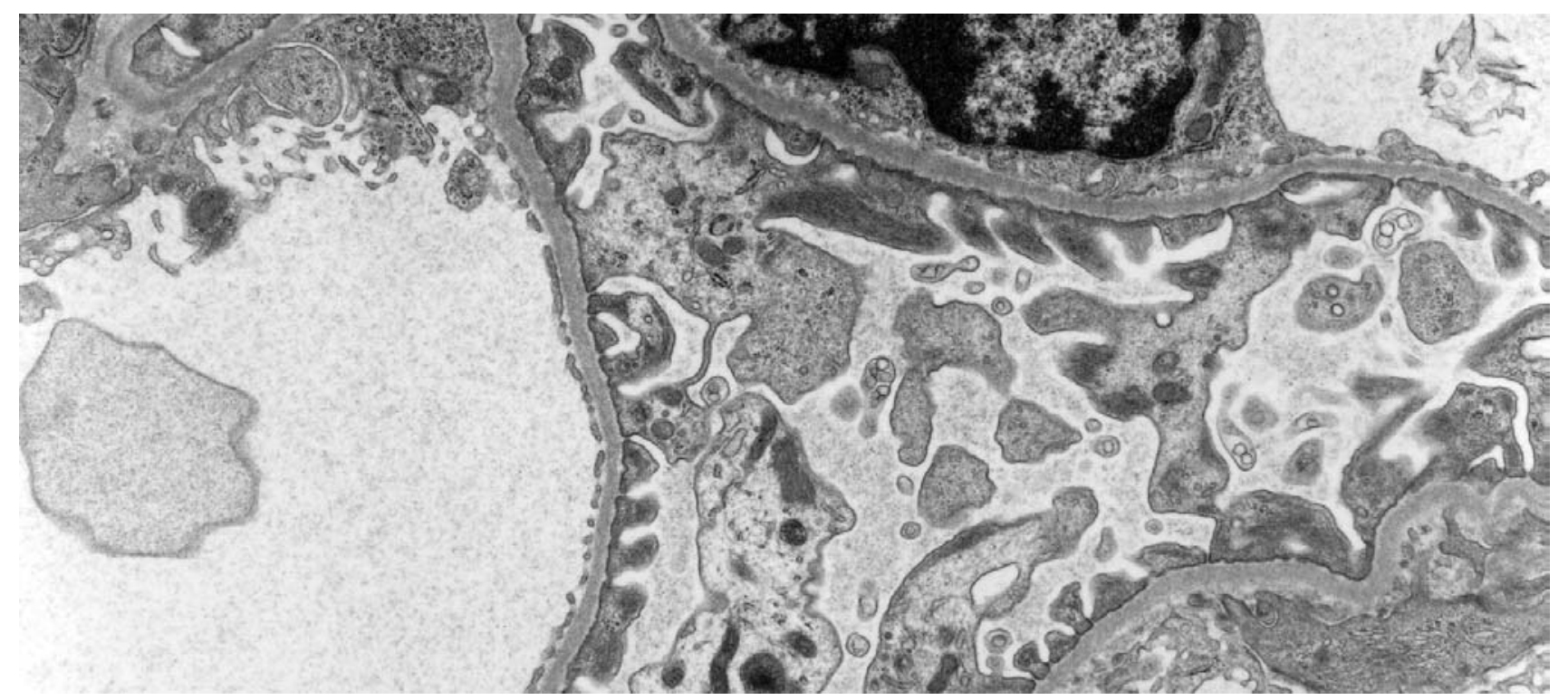

FIG. 1. Bench fixation of mouse kidney with Karnovsky's and osmium. 13,350X.

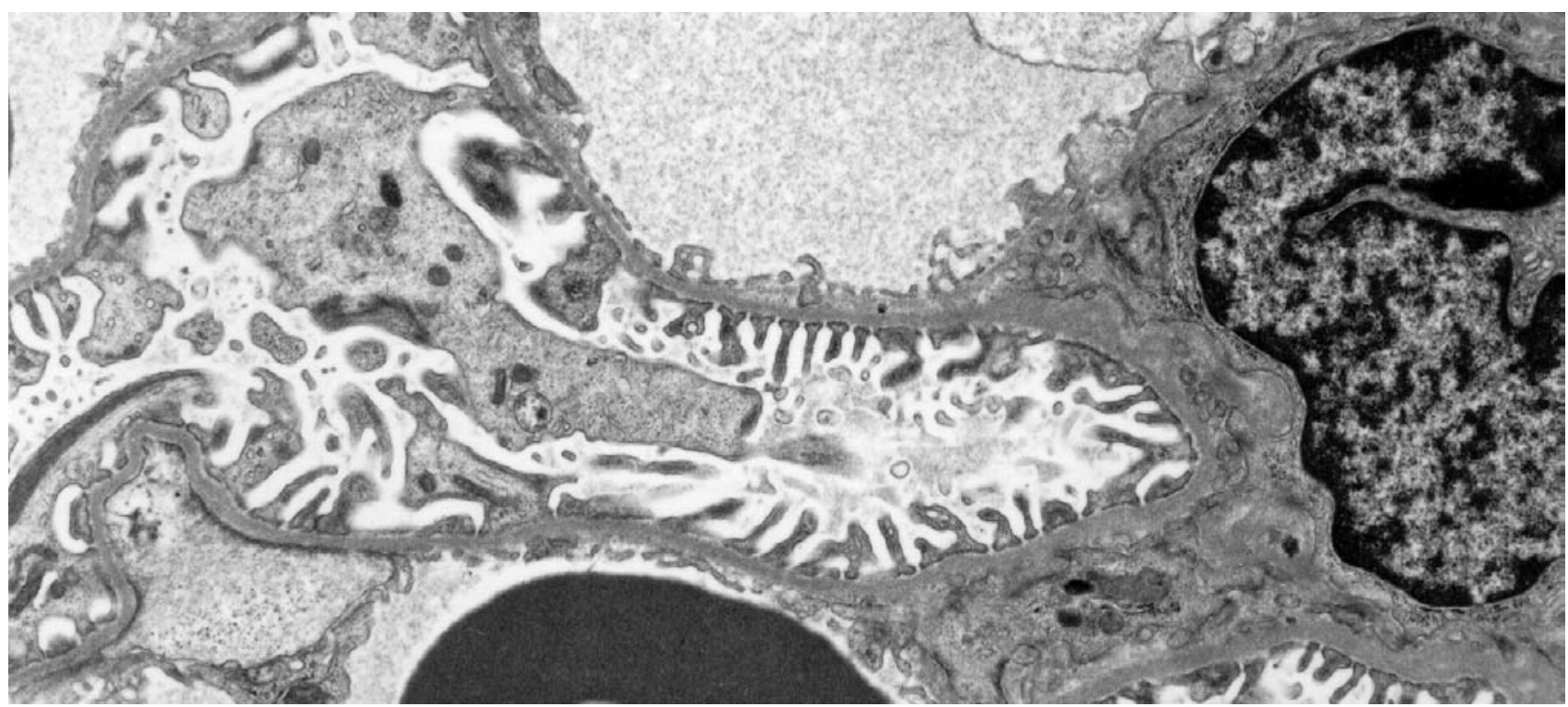

FIG. 2. Microwave-assisted fixation of mouse kidney with Karnovsky's and osmium. 13,350X. 